\title{
Load Frequency Control in Variable Inertia Systems
}

\author{
Mazheruddin H. Syed, Member, IEEE, Efren Guillo-Sansano, Member, IEEE, \\ Ali Mehrizi-Sani, Senior Member, IEEE, and Graeme M. Burt, Member, IEEE
}

\begin{abstract}
Conventional load frequency control primarily relies on large synchronous generation units to ensure regulation of the system frequency. However, its performance deteriorates as the system parameters, including inertia and droop coefficients, deviate from original system design. This letter proposes an augmented load frequency control (ALFC) to ensure robust frequency regulation under diurnal variations in system parameters that are expected in the future, renewables-rich power system. The superior performance of ALFC is demonstrated by several case studies, and its stability is assessed by small-signal analysis.
\end{abstract}

Index Terms-Converter, inertia, load frequency control.

\section{INTRODUCTION}

C ONVENTIONAL load frequency control (CLFC) was designed for operation within a legacy rigid power system where frequent changes in system parameters were limited. With the integration of renewables and decommissioning of conventional generating units, CLFC may no longer be considered suitable as it will need frequent tuning. To overcome this need, a tuningless direct load frequency controller was proposed in [1]. However, this approach is architecturally inflexible, requires system-wide observability, and does not ensure a locationally targeted remedial frequency control measure , i.e., provision of remedial frequency control measure close to the source of the disturbance. This latter merit is identified as a necessity to ensure stable operation in the future, renewables-rich grid [2]. In [3], a locationally targeted measure demonstrated robust temporal enhancement subject to system parameters change but exhibited deteriorated dynamic response in terms of increased overshoots.

This letter proposes an augmented load frequency control (ALFC), enabled by augmenting the CLFC with a modulated power balance control loop (MPBCL). The proposed power set point modulation with respect to the predicted dynamic trajectory of frequency addresses the shortcomings of [3] (which utilizes a balance control loop), yielding a dynamically robust performance. Therefore, ALFC ensures (i) a locationally targeted frequency response, (ii) tuning-free operation, and (iii) enhanced temporal and dynamic frequency response in the future renewables-rich power system.

\section{Conventional Load Frequency Control (CLFC)}

\section{A. The Approach}

Fig. 11 shows the load frequency control models of an interconnected power system of $M$-areas. The area system dynamics are

$$
\begin{aligned}
\Delta \dot{f}_{i}(t)= & \frac{1}{2 H_{i}}\left(-D \Delta f_{i}(t)+\Delta P_{m i}(t)+\Delta P_{i}^{r e n}(t)\right. \\
& \left.-\Delta P_{L i}(t)-\Delta P_{i}^{t i e}(t)+\Sigma \Delta P_{i}^{D S A}(t)\right) \\
\Delta \dot{P}_{m i}(t)= & \frac{1}{T_{b i}}\left(\Delta P_{g i}(t)+T_{a i} \Delta \dot{P}_{g i}(t)-\Delta P_{m i}(t)\right)
\end{aligned}
$$

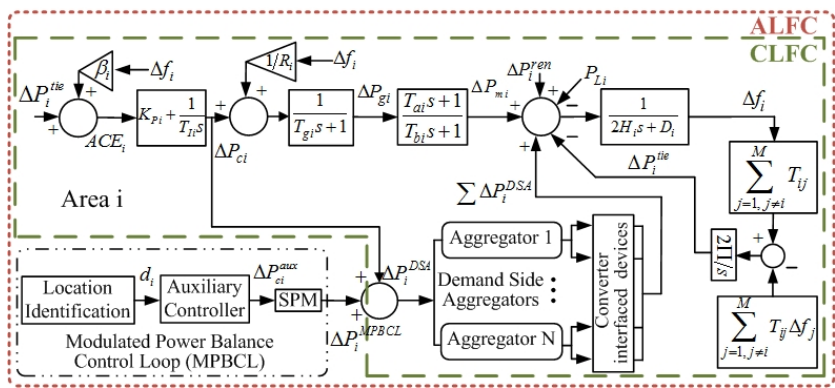

Fig. 1. Augmentation of CLFC with MPBCL enabling ALFC.

$$
\begin{aligned}
\Delta \dot{P}_{g i}(t) & =\frac{1}{T_{g i}}\left(p h a_{\mathrm{CGi}} \Delta P_{c i}(t)-\frac{1}{R_{i}} \Delta f_{i}(t)-\Delta P_{g i}(t)\right) \\
\Delta P_{i}^{\mathrm{tie}} & =2 \pi \int\left(\sum_{j=1, j \neq i}^{M} T_{i j}\left(\Delta f_{i}-\Delta f_{j}\right)\right) d t
\end{aligned}
$$

where $\Delta f_{i}$ is the frequency deviation of area $i$, and $H_{i}, D_{i}$, $R_{i}, T_{g i}, T_{a i}$ and $T_{b i}$ are the inertia constant, load damping coefficient, speed droop, governor and turbine time constants. $\Delta P_{c i}$ is the effort of the secondary control, $\Delta P_{m i}$ and $\Delta P_{g i}$ are the deviations of generator mechanical output and valve position, $\Delta P_{i}^{\mathrm{ren}}, \Delta P_{L i}, \Delta P_{i}^{\mathrm{tie}}$ and $\Delta P_{i}^{\mathrm{DSA}}$ are power variations of renewable generation, loads, tie-line, and demandside aggregators (DSA). $T_{i j}$ is the synchronizing torque. The secondary control input, area control error (ACE), is

$$
\operatorname{ACE}_{i}(t)=\beta_{i} \Delta f_{i}(t)+\Delta P_{i}^{\mathrm{tie}}(t)
$$

where $\beta_{i}$ is the frequency bias factor. A proportional integral (PI) control is used to regulate $\mathrm{ACE}$ (and hence $\Delta f_{i}$ ) to zero:

$$
\Delta P_{c i}(t)=-K_{P} \mathrm{ACE}_{i}(t)-\frac{1}{T_{I}} \int \mathrm{ACE}_{i}(t)
$$

\section{B. Challenges with the CLFC Approach}

The performance of CLFC is highly dependent on the system characteristics, such as inertia and droop. In modern power systems with high penetration of renewables, these parameters can have large diurnal variations [4]. To demonstrate the impact of parameter change, consider the response of CLFC for the five-area Great Britain (GB) power system's reducedorder model [3] subject to a disturbance emulated as a step increase in load, where $P$ is the size of disturbance. Figs. $2 \mathrm{a}$ $2 \mathrm{~b}$, and $2 \mathrm{c}$ show the results for nominal and off-nominal values of $H, R$, and $P$. With change in system parameters, CLFC responses exhibit increased overshoots and oscillations. CLFC offers no support for temporal or dynamic frequency response enhancement even with fast acting converter-interfaced demand-side resources.

\section{Augmented LoAd Frequency Control (ALFC)}

The conventional approach to frequency control focuses on the restoration of frequency to its nominal value through power regulation at the area boundary (as in Section II-A and [3]). 


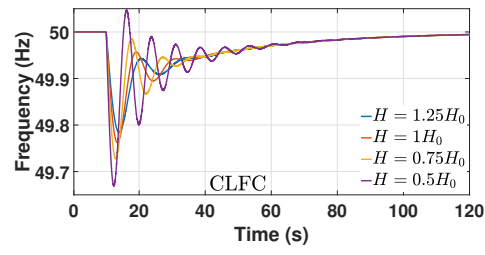

(a) $H$ variation, $R=R_{0}$ and $P=P_{0}$

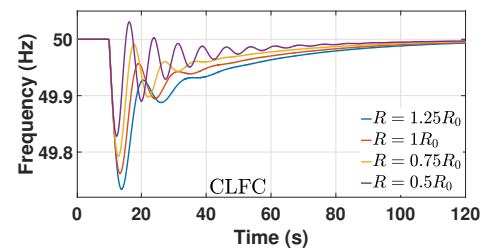

(b) $R$ variation, $H=H_{0}$ and $P=P_{0}$

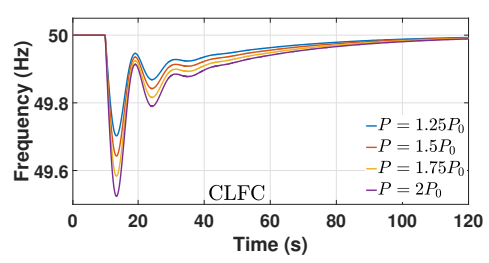

(c) $P$ variation, $H=H_{0}$ and $R=R_{0}$

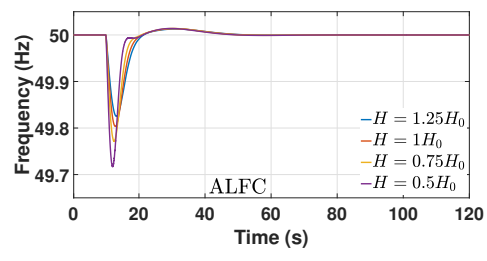

(d) $H$ variation, $R=R_{0}$ and $P=P_{0}$

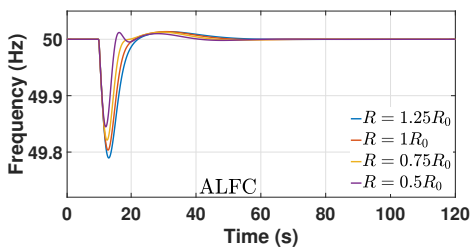

(e) $R$ variation, $H=H_{0}$ and $P=P_{0}$

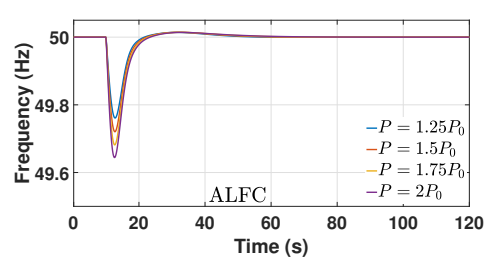

(f) $P$ variation, $H=H_{0}$ and $R=R_{0}$

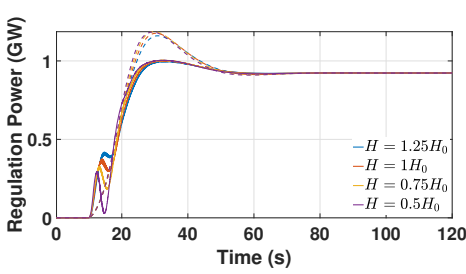

(g) $H$ variation, $R=R_{0}$ and $P=P_{0}$

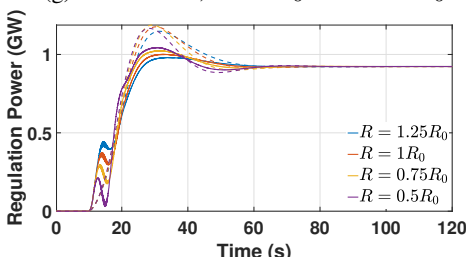

(h) $R$ variation, $H=H_{0}$ and $P=P_{0}$

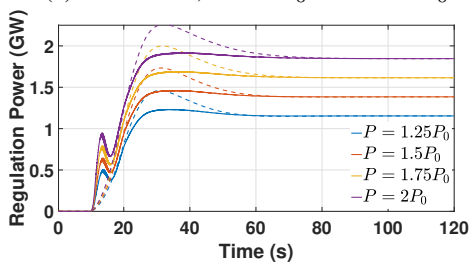

(i) $P$ variation, $H=H_{0}$ and $R=R_{0}$

Fig. 2. Performance evaluation of ALFC compared with CLFC.

The consequent dynamic trajectory of frequency is neglected. However, if the dynamic trajectory of frequency can be predicted and corresponding preemptive corrective adjustments to the power set point applied, the dynamic response of the system can be enhanced. With this premise, this letter proposes ALFC, the augmentation of CLFC with MPBCL shown in Fig. 11

\section{A. Location Identification and Auxiliary Controller}

To provide a locationally targeted response, MPBCL incorporates a location identification technique as proposed in [3], where $d_{i}=\Delta P_{i}^{\text {tie }}$ when the event is in area $i$ and 0 otherwise. Upon occurrence of an event in area $i, d_{i}$ is regulated to zero by a PI controller with its control effort represented as

$$
\Delta P_{c i}^{\text {aux }}(t)=-K_{\text {P,aux }} d_{i}(t)-\frac{1}{T_{\mathrm{I}, \text { aux }}} \int d_{i}(t)
$$

\section{B. Set Point Modulation (SPM)}

The output of the auxiliary controller is passed through a set point modulation (SPM) block, inspired by the approach proposed in [5] but further modified as follows:

- A linear look-ahead predictor is employed for its computational efficiency in real-time implementation.

- In distinction to the conventional SPM [5], the power set point is modulated using tracking error in frequency rather than power itself, as discussed in Section III-C.

The proposed control law is

$$
\Delta P_{i}^{\mathrm{MPBCL}}= \begin{cases}\Delta P_{c i}^{\mathrm{aux}}, & |\hat{e}(t)|<\varepsilon \\ \Delta P_{c i}^{\text {aux }}+m \hat{e}(t), & \text { otherwise }\end{cases}
$$

where $P_{c i}^{\text {aux }}$ is the power reference set point (Fig. 1,$P_{i}^{\mathrm{MPBCL}}$ is its modified set point, $m$ is the scaling factor, and $\hat{e}(t)$ is the predicted error calculated based on error defined as

$$
e(t)=f_{i}^{*}(t)-f_{i}(t)
$$

where $f_{i}^{*}$ and $f_{i}$ are the nominal and measured frequency respectively. The predictor calculates $\hat{e}(t)$ as

$$
\hat{e}(t)=e(t)+r(t) T_{\mathrm{p}}
$$

where $T_{p}$ is the prediction horizon and $r(t)$ is the average rate of change of the historical data based on least square error. With only one past data point, linear prediction yields $e(t)$ equal to the average of the past data point and the predicted term as

$$
\hat{e}(t)=2 e(t)-e\left(t-T_{\mathrm{p}}\right)
$$

Upon digression of $\hat{e}(t)$ beyond the deadband defined by tolerance threshold $\varepsilon$ as $[+\varepsilon,-\varepsilon]$, the set point is modified as per Eq. (8). The use of $\hat{e}(t)$ instead of $e(t)$ enables a preemptive correction yielding dynamic performance enhancement. Guidelines for parameter selection $\left(T_{p}\right.$ and $\left.m\right)$ in [5] remain applicable to the current application. The input to DSA is

$$
\Delta P_{i}^{\mathrm{DSA}}=\Delta P_{i}^{\mathrm{MPBCL}}+\Delta P_{c i}
$$

\section{Architectural Flexibility}

The network power-frequency characteristic (NPFC) [6] allows for the use of tracking error in frequency to modulate the power set point, with two distinct benefits:

1) NPFC defines a linear power-frequency relationship in the steady state. Direct incorporation of frequency measurement in SPM enables dynamic frequency response enhancement, which is not feasible if tracking error in power is utilized.

2) The use of error in frequency allows for architectural flexibility in implementation of the proposed approach. SPM can be implemented centrally at an area level by the system operator (as in Fig. 1. for all or a selected set of DSAs), or by the DSAs themselves within their operation centres (distributed) or at converter-interfaced demand side resources within their portfolio (decentralized), as long as frequency measurements are available. 


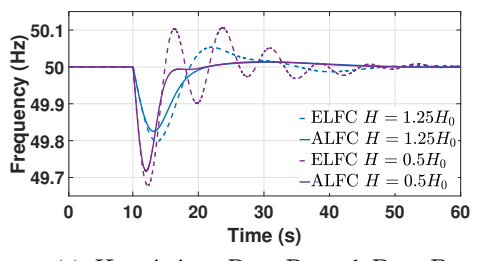

(a) $H$ variation, $R=R_{0}$ and $P=P_{0}$

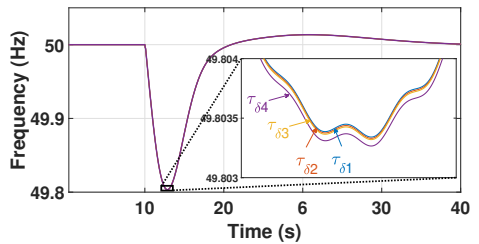

(d) ALFC performance with time delays

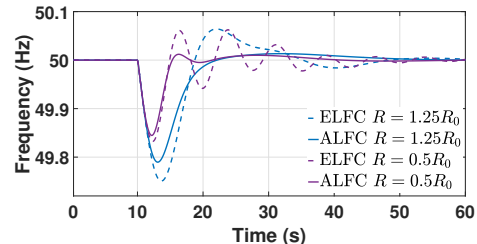

(b) $R$ variation, $H=H_{0}$ and $P=P_{0}$

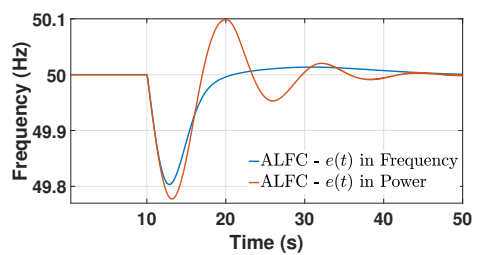

(e) Performance with different tracking errors

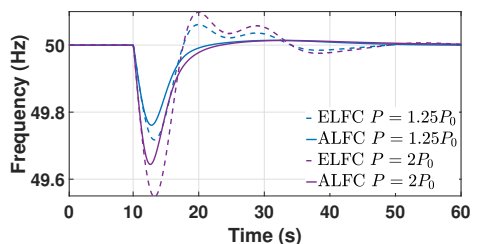

(c) $P$ variation, $H=H_{0}$ and $R=R_{0}$

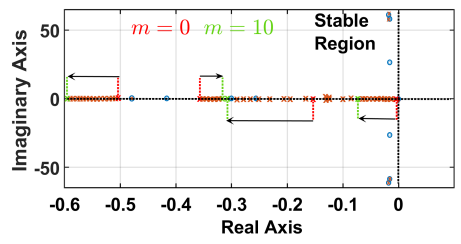

(f) Small-signal analysis of ALFC

Fig. 3. Extended performance evaluation of ALFC including small signal analysis.

\section{PERFormance EVAluation}

Studies are performed using the proposed method on the five-area GB power system with $H_{0}=4.23 \mathrm{~s}, R_{0}=0.13$, $P_{0}=1 \mathrm{GW}, K_{P}=0.1, T_{I}=75 \mathrm{~s}, K_{\mathrm{P}, \mathrm{aux}}=0.1, T_{\mathrm{I} \text {,aux }}=$ $5 \mathrm{~s}, m=10, \varepsilon=5 \mathrm{mHz}$. ALFC relies on existence of grid code-compliant, fast-acting converter-interfaced demandside resources within the portfolio of DSA. These have been modeled as first order equivalents that respond to a requested power command immediately with no additional response characteristics considered in this work [3].

\section{A. Simulation Case Studies}

Figs. 2d, 2e, and 2f show the system frequency response with ALFC for variation in system parameters. The input to SPM from the auxiliary controller (dotted lines) and the modulated output of MPBCL (solid lines) are shown in Figs. $2 \mathrm{~g}$, 2h, and $2 \mathrm{i}$. The ALFC yields tuning free operation, i.e., robust with respect to change in system parameters, requiring no re-tuning, with responses that (i) are well damped, (ii) have a reduced nadir, and (iii) have reduced overshoot. In addition, ALFC restores the frequency twice as fast compared with CLFC. This demonstrates the effective utilization of fast acting converter-interfaced demand side resources in the proposed approach. The performance of ALFC is also benchmarked against the enhanced load frequency control (ELFC) method proposed in [3], with the frequency responses of the system under varying system parameters shown in Figs. 3a, 3b and $3 \mathrm{c}$ In all cases, the ALFC exhibits superior performance with reduced nadir and reduced overshoot. Furthermore minimal impact is seen on the performance of ALFC from communications delays (Fig. 3d). This analysis assumes activation of DSA reserves through the Internet, with representative time delays as shown in Table I. In Fig. 3e, the distinctive design of SPM in comparison with the conventional approach in [5] is justified by the evidence of a poorer performance when tracking error in power is used.

\section{B. Small Signal Analysis}

The state-space model of the study system can be represented by $\dot{x}=A x+B u+F w, y=C x$; where $x=\left[x_{1}(t), x_{2}(t), x_{3}(t), x_{4}(t), x_{5}(t)\right]^{T}$ is the state vector, $u=\left[u_{1}(t), u_{2}(t)\right]^{T}$ is the control vector, and $w=\left[\Delta P_{L_{1}}(t), \ldots, \Delta P_{L_{M}}(t)\right]^{T}$ is the disturbance vector.
TABLE I

AVERAGE TIME DELAYS WITHIN GREAT BRITAIN [8

\begin{tabular}{|c|c|c|c|c|}
\hline & Cardiff $\left(\tau_{\delta 1}\right)$ & Coventry $\left(\tau_{\delta 2}\right)$ & Manchester $\left(\tau_{\delta 3}\right)$ & Newcastle $\left(\tau_{\delta 4}\right)$ \\
\hline Londor & $5.577 \mathrm{~ms}$ & $5.915 \mathrm{~ms}$ & $6.073 \mathrm{~ms}$ & $9.49 \mathrm{~ms}$ \\
\hline
\end{tabular}

The internal states are $x_{1}=\left[\Delta f_{1}, \ldots, \Delta f_{M}\right], x_{2}=$ $\left[\Delta P_{m_{1}}, \ldots, \Delta P_{m_{M}}\right], \quad x_{3}=\left[\Delta P_{1}^{t i e}, \ldots, \Delta P_{M}^{t i e}\right], \quad x_{4}=$ $\left[\int \mathrm{ACE}_{1} d t, \ldots, \int \mathrm{ACE}_{M} d t\right], x_{5}=\left[\int d_{1} d t, \ldots, \int d_{M} d t\right]$. The inputs are $u_{1}=\left[\Delta P_{c_{1}}, \ldots, \Delta P_{c_{M}}\right], \quad u_{2}=$ $\left[m \Delta P_{c 1}^{\text {aux }}, \ldots, m \Delta P_{c M}^{\text {aux }}\right]$. The coefficient matrices $A, B$, and $C$ are defined in [7]. The system is linearized around $f_{i}^{*}$ subject to reference disturbance $L_{0}$. Using MATLAB Simulink linear analysis toolbox, small signal analysis for value of $m$ changed from 0 to 10 (representing modulation up to chosen value of $\mathrm{m}$ ) in steps of 0.01 is undertaken. With bounded output $m \Delta P c i^{\text {aux }}$, the nonzero eigenvalues lie on the imaginary plane (Fig. 3f) demonstrating stable operation.

\section{Conclusions}

In this letter, an ALFC approach is proposed, its robustness to changes in system parameters is demonstrated, and smallsignal analysis is discussed. Two key features of the approach are (i) tuning-free operation under varying system conditions, and (ii) ease of integration and enhanced scalability given its architectural flexibility facilitating the move towards an inverter dominated grid.

\section{REFERENCES}

[1] A. M. Prostejovsky, M. Marinelli, M. Rezkalla et al., "Tuningless load frequency control through active engagement of distributed resources," IEEE Trans. Power Syst., vol. 33, no. 3, pp. 2929-2939, May 2018.

[2] V. V. Terzija, "Adaptive underfrequency load shedding based on the magnitude of the disturbance estimation," IEEE Trans. Power Syst., vol. 21, no. 3, pp. 1260-1266, Aug 2006.

[3] M. H. Syed, E. Guillo-Sansano, S. M. Blair et al., "Enhanced load frequency control: incorporating locational information for temporal enhancement," IET GTD, vol. 13, no. 10, pp. 1865-1874, 2019.

[4] B. Kroposki, B. Johnson, Y. Zhang et al., "Achieving a 100\% Renewable Grid," IEEE Power and Energy Mag., vol. 15, no. 2, pp. 61-73, 2017.

[5] M. Yazdanian, A. Mehrizi-Sani, R. Seebacher et al., "Smooth reference modulation to improve dynamic response in drive systems," IEEE Trans. Power Electron., vol. 33, no. 7, pp. 6434-6443, Jul. 2018.

[6] Policy P1: Load-Frequency Control and Performance. in Continental Europe Operation Handbook, ENTSO-E.

[7] N. V. Ramana, Power System Operation and Control. India: Pearson Education, 2011.

[8] "Global Ping Statistics," https://wondernetwork.com/pings Accessed: 2020-04-09. 